\title{
A New Coupled Fractional Reduced Differential Transform Method for the Numerical Solutions of $(2+1)$-Dimensional Time Fractional Coupled Burger Equations
}

\author{
S. Saha Ray \\ Department of Mathematics, National Institute of Technology, Rourkela 769008, India \\ Correspondence should be addressed to S. Saha Ray; santanusaharay@yahoo.com
}

Received 16 July 2013; Accepted 26 February 2014; Published 13 May 2014

Academic Editor: Antonio Munjiza

Copyright (C) 2014 S. Saha Ray. This is an open access article distributed under the Creative Commons Attribution License, which permits unrestricted use, distribution, and reproduction in any medium, provided the original work is properly cited.

A very new technique, coupled fractional reduced differential transform, has been implemented to obtain the numerical approximate solution of $(2+1)$-dimensional coupled time fractional burger equations. The fractional derivatives are described in the Caputo sense. By using the present method we can solve many linear and nonlinear coupled fractional differential equations. The obtained results are compared with the exact solutions. Numerical solutions are presented graphically to show the reliability and efficiency of the method.

\section{Introduction}

In the past decades, the fractional differential equations have been widely used in various fields of applied science and engineering [1-10]. Fractional calculus has been used to model physical and engineering processes that are found to be best described by fractional differential equations. For that reason we need a reliable and efficient technique for the solution of fractional differential equations. An immense effort has been expended over the last many years to find robust and efficient numerical and analytical methods for solving such fractional differential equations. In the present analysis, a new approximate numerical technique, coupled fractional reduced differential transform method (CFRDTM), has been proposed which is applicable for coupled fractional differential equations. The proposed method is a very powerful solver for linear and nonlinear coupled fractional differential equations. It is relatively a new approach to provide the solution very efficiently and accurately.

The Burgers model of turbulence is a very important fluid dynamic model and the study of this model and the theory of shock waves have been considered by many authors both to obtain conceptual understanding of a class of physical flows and for testing various numerical methods. The study of coupled Burgers equations is very significant for the system is a simple model of sedimentation or evolution of scaled volume concentrations of two kinds of particles in fluid suspensions or colloids, under the effect of gravity [11].

In this paper, $(2+1)$-dimensional coupled time fractional burger equations have been considered. The paper is organized as follows. In Section 2, a brief review of the theory of fractional calculus has been provided for precise purpose of this paper. In Section 3, the coupled fractional reduced differential transform method has been analyzed in detail. In Section 4, CFRDTM has been applied to determine the approximate solutions for the coupled time fractional burger equations. The obtained results show the efficiency and simplicity of the proposed method. Finally, conclusions are presented.

\section{Mathematical Preliminaries of Fractional Calculus}

The fractional calculus was first anticipated by Leibnitz, one of the founders of standard calculus, in a letter written in 1695. This calculus involves different definitions of the fractional operators as well as the Riemann-Liouville fractional derivative, Caputo derivative, Riesz derivative, and GrunwaldLetnikov fractional derivative [1]. The fractional calculus 
has gained considerable importance during the past decades mainly due to its applications in diverse fields of science and engineering. For the purpose of this paper Caputo's definition of fractional derivative will be used, taking the advantage of Caputo's approach that the initial conditions for fractional differential equations with Caputo's derivatives take on the traditional form as for integer-order differential equations.

2.1. Definition of Riemann-Liouville Integral. The most frequently encountered definition of an integral of fractional order is the Riemann-Liouville integral [1], in which the fractional integral of order $\alpha(>0)$ is defined as

$$
J^{\alpha} f(t)=\frac{1}{\Gamma(\alpha)} \int_{0}^{t}(t-\tau)^{\alpha-1} f(\tau) d \tau, \quad t>0, \alpha \in \mathbf{R}^{+},
$$

where $\mathbf{R}^{+}$is the set of positive real numbers.

2.2. Definition of Caputo Fractional Derivative. The fractional derivative, introduced by Caputo $[12,13]$ in the late sixties, is called Caputo Fractional Derivative. The fractional derivative of $f(t)$ in the Caputo sense is defined by

$$
\begin{aligned}
D_{t}^{\alpha} f(t) & =J^{m-\alpha} D^{m} f(t) \\
& =\left\{\begin{array}{l}
\frac{1}{\Gamma(m-\alpha)} \int_{0}^{t}(t-\tau)^{(m-\alpha-1)} \frac{d^{m} f(\tau)}{d \tau^{m}} d \tau, \\
\text { if } m-1<\alpha<m, m \in N \\
\frac{d^{m} f(t)}{d t^{m}}, \\
\text { if } \alpha=m, m \in N,
\end{array}\right.
\end{aligned}
$$

where the parameter $\alpha$ is the order of the derivative and is allowed to be real or even complex. In this paper only real and positive $\alpha$ will be considered.

For the Caputo's derivative we have

$$
\begin{aligned}
& D^{\alpha} C=0, \quad(C \text { is a constant }) \\
& D^{\alpha} t^{\beta}= \begin{cases}0, & \beta \leq \alpha-1 \\
\frac{\Gamma(\beta+1) t^{\beta-\alpha}}{\Gamma(\beta-\alpha+1)}, & \beta>\alpha-1 .\end{cases}
\end{aligned}
$$

Being similar to integer-order differentiation, Caputo's derivative is linear:

$$
D^{\alpha}(\gamma f(t)+\delta g(t))=\gamma D^{\alpha} f(t)+\delta D^{\alpha} g(t),
$$

where $\gamma$ and $\delta$ are constants and satisfy so called Leibnitz's rule:

$$
D^{\alpha}(g(t) f(t))=\sum_{k=0}^{\infty}\left(\begin{array}{l}
\alpha \\
k
\end{array}\right) g^{(k)}(t) D^{\alpha-k} f(t),
$$

where $f(\tau)$ is continuous in $[0, t]$ and $g(\tau)$ has $n+1$ continuous derivatives in $[0, t]$.

Lemma 1. If $m-1<\alpha<m, m \in N$, then

$$
\begin{aligned}
& D^{\alpha} J^{\alpha} f(t)=f(t), \\
& J^{\alpha} D^{\alpha} f(t)=f(t)-\sum_{k=0}^{m-1} \frac{t^{k}}{k !} f^{(k)}(0+), \quad t>0 .
\end{aligned}
$$

Theorem 2 (generalized Taylor's formula; see [14]). Suppose that $D_{a}^{k \alpha} f(t) \in C(a, b]$ for $k=0,1, \ldots, n+1$, where $0<\alpha \leq 1$; we have

$$
f(t)=\sum_{i=0}^{n} \frac{(t-a)^{i \alpha}}{\Gamma(i \alpha+1)}\left[D_{a}^{i \alpha} f(t)\right]_{t=a}+\mathfrak{R}_{n}^{\alpha}(t ; a),
$$

with $\mathfrak{R}_{n}^{\alpha}(t ; a)=\left((t-a)^{(n+1) \alpha} / \Gamma((n+1) \alpha+1)\right)\left[D_{a}^{(n+1) \alpha} f(t)\right]_{t=\xi}$, $a \leq \xi \leq t, \forall t \in(a, b]$, where $D_{a}^{k \alpha}=D_{a}^{\alpha} \cdot D_{a}^{\alpha} \cdot D_{a}^{\alpha} \cdots D_{a}^{\alpha}(k$ times).

\section{Coupled Fractional Reduced Differential Transform Method (CFRDTM)}

In order to introduce coupled fractional reduced differential transform, $U(h, k-h)$ is considered as the coupled fractional reduced differential transform of $u(x, y, t)$. If function $u(x, y, t)$ is analytic and differentiated continuously with respect to time $t$, then we define the fractional coupled reduced differential transform of $u(x, y, t)$ as

$$
\begin{aligned}
U(h, k-h)= & \frac{1}{\Gamma(h \alpha+(k-h) \beta+1)} \\
& \times\left[D_{t}^{(h \alpha+(k-h) \beta)} u(x, y, t)\right]_{t=0},
\end{aligned}
$$

whereas the inverse transform of $U(h, k-h)$ is

$$
u(x, y, t)=\sum_{k=0}^{\infty} \sum_{h=0}^{k} U(h, k-h) t^{h \alpha+(k-h) \beta}
$$

which is one of the solutions of coupled fractional differential equations.

Theorem 3. Suppose that $U(h, k-h), V(h, k-h)$, and $W(h, k-$ $h)$ are the coupled fractional reduced differential transform of the functions $u(x, y, t), v(x, y, t)$, and $w(x, y, t)$, respectively.

(i) If $u(x, y, t)=f(x, y, t) \pm g(x, y, t)$, then $U(h, k-h)=$ $F(h, k-h) \pm G(h, k-h)$.

(ii) If $u(x, y, t)=a f(x, y, t)$, where $a \in \mathbf{R}$, then $U(h, k-$ $h)=a F(h, k-h)$.

(iii) If $f(x, y, t)=u(x, y, t) v(x, y, t)$, then $F(h, k-h)=$ $\sum_{l=0}^{h} \sum_{s=0}^{k-h} U(h-l, s) V(l, k-h-s)$.

(iv) If $f(x, y, t)=D_{t}^{\alpha} u(x, y, t)$, then

$F(h, k-h)=\frac{\Gamma((h+1) \alpha+(k-h) \beta+1)}{\Gamma(h \alpha+(k-h) \beta+1)} U(h+1, k-h)$.

(v) If $f(x, y, t)=D_{t}^{\beta} v(x, y, t)$, then

$$
F(h, k-h)=\frac{\Gamma(h \alpha+(k-h+1) \beta+1)}{\Gamma(h \alpha+(k-h) \beta+1)} V(h, k-h+1) .
$$




\section{Application of CFRDTM for the Solutions for $(2+1)$-Dimensional Time Fractional Coupled Burgers Equations}

Example 4. Consider the following $(2+1)$-dimensional time fractional coupled Burgers equations [15]:

$$
\begin{aligned}
& D_{t}^{\alpha} u+u \frac{\partial u}{\partial x}+v \frac{\partial u}{\partial y}=\frac{1}{R}\left(\frac{\partial^{2} u}{\partial x^{2}}+\frac{\partial^{2} u}{\partial y^{2}}\right), \\
& D_{t}^{\beta} v+u \frac{\partial v}{\partial x}+v \frac{\partial v}{\partial y}=\frac{1}{R}\left(\frac{\partial^{2} v}{\partial x^{2}}+\frac{\partial^{2} v}{\partial y^{2}}\right),
\end{aligned}
$$

subject to the initial conditions

$$
\begin{aligned}
& u(x, y, 0)=\frac{3}{4}-\frac{1}{4(1+\exp ((R / 32)(-4 x+4 y)))}, \\
& v(x, y, 0)=\frac{3}{4}+\frac{1}{4(1+\exp ((R / 32)(-4 x+4 y)))},
\end{aligned}
$$

where $0 \leq x, y \leq 1, t>0,0<\alpha, \beta \leq 1$, and $R$ is the Reynolds number.

The exact solutions of (13) and (14), for the special case where $\alpha=\beta=1$, are given by

$$
\begin{aligned}
& u(x, y, t)=\frac{3}{4}-\frac{1}{4(1+\exp ((R / 32)(-4 x+4 y-t)))}, \\
& v(x, y, t)=\frac{3}{4}+\frac{1}{4(1+\exp ((R / 32)(-4 x+4 y-t)))}
\end{aligned}
$$

In order to assess the advantages and the accuracy of the CFRDTM, we consider the $(2+1)$-dimensional time fractional coupled burgers equations. Firstly, we derive the recursive formula from (13) and (14). Now, $U(h, k-h)$ and $V(h, k-h)$ are considered as the coupled fractional reduced differential transform of $u(x, y, t)$ and $v(x, y, t)$, respectively, where $u(x, y, t)$ and $v(x, y, t)$ are the solutions of coupled fractional differential equations. Here, $U(0,0)=u(x, y, 0)$, $V(0,0)=v(x, y, 0)$ are the given initial conditions. Without loss of generality, the following assumptions have been taken:

$$
\begin{gathered}
U(0, j)=0, \quad j=1,2,3, \ldots, \\
V(i, 0)=0, \quad i=1,2,3, \ldots
\end{gathered}
$$

Applying CFRDTM to (13), we obtain the following recursive formula:

$$
\begin{gathered}
\frac{\Gamma((h+1) \alpha+(k-h) \beta+1)}{\Gamma(h \alpha+(k-h) \beta+1)} U(h+1, k-h) \\
=\frac{\partial^{2}}{\partial x^{2}} U(h, k-h)+\frac{\partial}{\partial x}
\end{gathered}
$$

$$
\begin{aligned}
& \times\left(\sum_{l=0}^{h} \sum_{s=0}^{k-h} U(h-l, s) U(l, k-h-s)\right) \\
& -\frac{\partial}{\partial x}\left(\sum_{l=0}^{h} \sum_{s=0}^{k-h} U(h-l, s) V(l, k-h-s)\right) .
\end{aligned}
$$

From the initial condition of (15), we have

$$
U(0,0)=u(x, y, 0)
$$

In the same manner, we can obtain the following recursive formula from (14):

$$
\begin{aligned}
& \frac{\Gamma(h \alpha+(k-h+1) \beta+1)}{\Gamma(h \alpha+(k-h) \beta+1)} V(h, k-h+1) \\
& =\frac{\partial^{2}}{\partial x^{2}} V(h, k-h)+\frac{\partial}{\partial x} \\
& \quad \times\left(\sum_{l=0}^{h} \sum_{s=0}^{k-h} V(l, k-h-s) V(h-l, s)\right) \\
& -\frac{\partial}{\partial x}\left(\sum_{l=0}^{h} \sum_{s=0}^{k-h} U(l, k-h-s) V(h-l, s)\right) .
\end{aligned}
$$

From the initial condition of (16), we have

$$
V(0,0)=v(x, y, 0) \text {. }
$$

According to CFRDTM, using recursive equation (19) with initial condition (20) and also using recursive scheme (21) with initial condition (22) simultaneously, we obtain

$$
\begin{gathered}
U(1,0)=-\frac{\exp ((R / 8)(x-y)) R}{128(1+\exp ((R / 8)(x-y)))^{2} \Gamma(1+\alpha)}, \\
V(0,1)=-\frac{\exp ((R / 8)(x-y)) R}{128(1+\exp ((R / 8)(x-y)))^{2} \Gamma(1+\beta)}, \\
U(1,1)=-\frac{\exp ((R / 4)(x-y)) R^{2}}{4096(1+\exp ((R / 8)(x-y)))^{4} \Gamma(1+\alpha+\beta)},
\end{gathered}
$$

$V(0,2)$

$$
\begin{aligned}
=-\left(\exp \left(\frac{R}{8}(x-y)\right)\right. \\
\times\left(-1+\exp \left(\frac{R}{8}(x-y)\right)\right. \\
\left.\left.+\exp \left(\frac{R}{4}(x-y)\right)\right) R^{2}\right) \\
\times\left(4096\left(1+\exp \left(\frac{R}{8}(x-y)\right)\right)^{4} \Gamma(1+2 \beta)\right)^{-1},
\end{aligned}
$$


$U(2,0)$

$$
\begin{aligned}
& =\left(\exp \left(\frac{R}{8}(x-y)\right)\right. \\
& \times\left(-1+\exp \left(\frac{R}{8}(x-y)\right)\right. \\
& \left.\left.+\exp \left(\frac{R}{4}(x-y)\right)\right) R^{2}\right) \\
& \times\left(4096\left(1+\exp \left(\frac{R}{8}(x-y)\right)\right)^{4} \Gamma(1+2 \alpha)\right)^{-1}, \\
& V(1,1)=\frac{\exp ((R / 4)(x-y)) R^{2}}{4096(1+\exp ((R / 8)(x-y)))^{4} \Gamma(1+\alpha+\beta)}, \\
& =\left(\exp \left(\frac{R}{4}(x-y)\right)\right. \\
& \times\left(-1+\exp \left(\frac{R}{8}(x-y)\right)\right. \\
& \left.\left.+\exp \left(\frac{R}{4}(x-y)\right)\right) R^{3}\right) \\
& \times\left(131072\left(1+\exp \left(\frac{R}{8}(x-y)\right)\right)^{6} \Gamma(1+\alpha+2 \beta)\right)^{-1},
\end{aligned}
$$

and so on.

The approximate solutions, obtained in the series form, are given by

$$
\begin{aligned}
& u(x, y, t) \\
& =\sum_{k=0}^{\infty} \sum_{h=0}^{k} U(h, k-h) t^{(h \alpha+(k-h) \beta)} \\
& =U(0,0)+\sum_{k=1}^{\infty} \sum_{h=1}^{k} U(h, k-h) t^{(h \alpha+(k-h) \beta)} \\
& =\frac{3}{4}-\frac{1}{4(1+\exp ((R / 32)(-4 x+4 y-t)))} \\
& -\frac{\exp ((R / 8)(x-y)) R t^{\alpha}}{128(1+\exp ((R / 8)(x-y)))^{2} \Gamma(1+\alpha)} \\
& +\left(\exp \left(\frac{R}{8}(x-y)\right)\right. \\
& \quad \times\left(-1+\exp \left(\frac{R}{8}(x-y)\right)\right. \\
& \quad+\left(4096\left(1+\exp \left(\frac{R}{4}(x-y)\right)\right) R^{2} t^{2 \alpha}\right)
\end{aligned}
$$

$$
\begin{aligned}
& -\left(\exp \left(\frac{R}{4}(x-y)\right) R^{2} t^{\alpha+\beta}\right) \\
& \times\left(4096\left(1+\exp \left(\frac{R}{8}(x-y)\right)\right)^{4} \Gamma(1+\alpha+\beta)\right)^{-1} \\
& +\cdots \\
v(x, y, t) & \\
= & \sum_{k=0}^{\infty} \sum_{h=0}^{k} V(h, k-h) t^{(h \alpha+(k-h) \beta)} \\
= & V(0,0)+\sum_{k=1}^{\infty} \sum_{h=0}^{k} V(h, k-h) t^{(h \alpha+(k-h) \beta)} \\
= & \frac{3}{4}+\frac{1}{4(1+\exp ((R / 32)(-4 x+4 y)))} \\
& -\frac{\exp ((R / 8)(x-y)) R t^{\beta}}{128(1+\exp ((R / 8)(x-y)))^{2} \Gamma(1+\beta)} \\
& +\frac{\exp ((R / 4)(x-y)) R^{2} t^{\alpha+\beta}}{4096(1+\exp ((R / 8)(x-y)))^{4} \Gamma(1+\alpha+\beta)} \\
& +\cdots \\
& +\left(\exp \left(\frac{R}{8}(x-y)\right)\right. \\
& \times\left(-1+\exp \left(\frac{R}{8}(x-y)\right)\right. \\
& \left.\left.+\exp \left(\frac{R}{4}(x-y)\right)\right) R^{2} t^{2 \beta}\right) \\
& \\
& \\
& \\
& \\
& \\
&
\end{aligned}
$$

In special case for $\alpha=1$ and $\beta=1$, the solutions in (24) coincide exactly with the Taylor series expansion of the exact solutions in (17)

$$
\begin{aligned}
u(x, y, t) & \\
= & \frac{3}{4}-\frac{1}{4(1+\exp ((R / 32)(-4 x+4 y)))} \\
& \quad-\frac{\exp ((R / 8)(x+y)) R t}{128(\exp (R x / 8)+\exp (R y / 8))^{2}} \\
& +\frac{\exp ((R / 8)(x+y))(\exp (R x / 8)-\exp (R y / 8)) R^{2} t^{2}}{8192(\exp (R x / 8)+\exp (R y / 8))^{3}} \\
& +\cdots, \\
v(x, & y, t) \\
= & \frac{3}{4}+\frac{1}{4(1+\exp ((R / 32)(-4 x+4 y)))}
\end{aligned}
$$




$$
\begin{aligned}
& +\frac{\exp ((R / 8)(x+y)) R t}{128(\exp (R x / 8)+\exp (R y / 8))^{2}} \\
& +\left(\exp \left(\frac{R}{8}(x+y)\right)\right. \\
& \left.\quad \times\left(-\exp \left(\frac{R x}{8}\right)+\exp \left(\frac{R y}{8}\right)\right) R^{2} t^{2}\right) \\
& \times\left(8192\left(\exp \left(\frac{R x}{8}\right)+\exp \left(\frac{R y}{8}\right)\right)^{3}\right)^{-1}+\cdots .
\end{aligned}
$$

In case of $\alpha=1$ and $\beta=1$, Table 1 cites the comparison of results obtained in proposed CRFDTM with variational iteration method (VIM) when $y=1$. From these results we can certainly conclude that the proposed method CRFDTM provides remarkable accuracy in comparison to VIM. In the computation of Table 1 , the value of $R$ has been taken as 100 and the numerical approximate solutions for $u(x, y, t)$ and $v(x, y, t)$ have been evaluated with four terms in CRFDTM and consequently compared with third approximations in VIM.

Example 5. Consider the following $(2+1)$-dimensional time fractional coupled Burgers equations [16]:

$$
\begin{aligned}
& D_{t}^{\alpha} u-\frac{\partial^{2} u}{\partial x^{2}}-\frac{\partial^{2} u}{\partial y^{2}}-2 u \frac{\partial u}{\partial x}-2 v \frac{\partial u}{\partial y}=0 \\
& D_{t}^{\beta} v-\frac{\partial^{2} v}{\partial x^{2}}-\frac{\partial^{2} v}{\partial y^{2}}-2 u \frac{\partial v}{\partial x}-2 v \frac{\partial v}{\partial y}=0
\end{aligned}
$$

subject to the initial conditions

$$
\begin{gathered}
u(x, y, 0)=1-\tanh (-x+2 y+1) \\
v(x, y, 0)=1+2 \tanh (-x+2 y+1)
\end{gathered}
$$

First, we derive the recursive formula from (26) and (27). Now, $U(h, k-h)$ and $V(h, k-h)$ are considered as the coupled fractional reduced differential transform of $u(x, t)$ and $v(x, t)$, respectively, where $u(x, t)$ and $v(x, t)$ are the solutions of coupled fractional differential equations. Here, $U(0,0)=$ $u(x, 0), V(0,0)=v(x, 0)$ are the given initial conditions. Without loss of generality, the following assumptions have been taken:

$$
\begin{gathered}
U(0, j)=0, \quad j=1,2,3, \ldots, \\
V(i, 0)=0, \quad i=1,2,3, \ldots
\end{gathered}
$$

Applying CFRDTM to (26), we obtain the following recursive formula:

$$
\begin{aligned}
& \frac{\Gamma((h+1) \alpha+(k-h) \beta+1)}{\Gamma(h \alpha+(k-h) \beta+1)} U(h+1, k-h) \\
& =\frac{\partial^{2}}{\partial x^{2}} U(h, k-h) \\
& \quad+\frac{\partial}{\partial x}\left(\sum_{l=0}^{h} \sum_{s=0}^{k-h} U(h-l, s) U(l, k-h-s)\right) \\
& \quad-\frac{5}{2} \frac{\partial}{\partial x}\left(\sum_{l=0}^{h} \sum_{s=0}^{k-h} U(h-l, s) V(l, k-h-s)\right) .
\end{aligned}
$$

From the initial condition of (28), we have

$$
U(0,0)=u(x, y, 0)
$$

In the same manner, we can obtain the following recursive formula from (27):

$$
\begin{aligned}
& \frac{\Gamma(h \alpha+(k-h+1) \beta+1)}{\Gamma(h \alpha+(k-h) \beta+1)} V(h, k-h+1) \\
& =\frac{\partial^{2}}{\partial x^{2}} V(h, k-h) \\
& \quad+\frac{\partial}{\partial x}\left(\sum_{l=0}^{h} \sum_{s=0}^{k-h} V(l, k-h-s) V(h-l, s)\right) \\
& \quad-\frac{5}{2} \frac{\partial}{\partial x}\left(\sum_{l=0}^{h} \sum_{s=0}^{k-h} U(l, k-h-s) V(h-l, s)\right) .
\end{aligned}
$$

From the initial condition of (29), we have

$$
V(0,0)=v(x, y, 0) \text {. }
$$

According to CFRDTM, using recursive equation (31) with initial condition (32) and also using recursive scheme (33) with initial condition (34) simultaneously, we obtain successively

$$
\begin{aligned}
& U(1,0)=-\frac{2 \operatorname{sech}^{2}(1-x+2 y)}{\Gamma(1+\alpha)} \\
& V(0,1)=\frac{4 \operatorname{sech}^{2}(1-x+2 y)}{\Gamma(1+\beta)} \\
& U(1,1)=-\frac{16 \operatorname{sech}^{4}(1-x+2 y)}{\Gamma(1+\alpha+\beta)}
\end{aligned}
$$


TABLE 1: The comparison of results obtained in proposed method with VIM when $y=1, \alpha=1$, and $\beta=1$.

\begin{tabular}{|c|c|c|c|c|c|}
\hline & $x$ & $\left|u_{\text {Exact }}-u_{\text {VIM }}\right|$ & $\left|v_{\text {Exact }}-v_{\mathrm{VIM}}\right|$ & $\left|u_{\text {Exact }}-u_{\text {CRFDTM }}\right|$ & $\left|v_{\text {Exact }}-v_{\text {CRFDTM }}\right|$ \\
\hline \multirow{5}{*}{$t=0.1$} & 0.1 & $2.1 E-9$ & $0.8 E-9$ & $8.51241 E-11$ & $1.37701 E-9$ \\
\hline & 0.2 & $6.9 E-9$ & $4.3 E-9$ & $2.96787 E-10$ & $4.80359 E-9$ \\
\hline & 0.3 & $1.62 E-8$ & $1.69 E-8$ & $1.03193 E-9$ & $1.67338 E-8$ \\
\hline & 0.4 & $5.97 E-8$ & $5.48 E-8$ & $3.55374 E-9$ & $5.80129 E-8$ \\
\hline & 0.5 & $1.866 E-7$ & $1.886 E-7$ & $1.18268 E-8$ & $1.97734 E-7$ \\
\hline \multirow{5}{*}{$t=0.2$} & 0.1 & $2.44 E-8$ & $2.25 E-8$ & $2.87822 E-9$ & $2.35485 E-8$ \\
\hline & 0.2 & $8.31 E-8$ & $8.08 E-8$ & $1.00343 E-8$ & $8.21431 E-8$ \\
\hline & 0.3 & $2.85 E-7$ & $2.858 E-7$ & $3.48807 E-8$ & $2.8611 E-7$ \\
\hline & 0.4 & $9.794 E-7$ & $9.745 E-7$ & $1.20019 E-7$ & $9.91365 E-7$ \\
\hline & 0.5 & $3.2012 E-6$ & $3.2026 E-6$ & $3.98184 E-7$ & $3.37271 E-6$ \\
\hline \multirow{5}{*}{$t=0.3$} & 0.1 & $1.288 E-7$ & $1.276 E-7$ & $2.31437 E-8$ & $1.27787 E-7$ \\
\hline & 0.2 & $4.470 E-7$ & $4.441 E-7$ & $8.06788 E-8$ & $4.4573 E-7$ \\
\hline & 0.3 & $1.5458 E-6$ & $1.5464 E-6$ & $2.8037 E-7$ & $1.55222 E-6$ \\
\hline & 0.4 & $5.3028 E-5$ & $5.2976 E-6$ & $9.63714 E-7$ & $5.3749 E-6$ \\
\hline & 0.5 & $1.73542 E-5$ & $1.73552 E-5$ & $3.18549 E-6$ & 0.000018244 \\
\hline \multirow{5}{*}{$t=0.4$} & 0.1 & $4.351 E-6$ & $4.336 E-6$ & $1.03504 E-7$ & $4.34228 E-7$ \\
\hline & 0.2 & $1.5143 E-6$ & $1.5114 E-6$ & $3.60779 E-7$ & $1.51452 E-6$ \\
\hline & 0.3 & $5.2532 E-6$ & $5.2531 E-6$ & $1.25332 E-6$ & $5.27299 E-6$ \\
\hline & 0.4 & $1.80073 E-5$ & $1.80029 E-5$ & $4.30269 E-6$ & 0.0000182442 \\
\hline & 0.5 & $5.88677 E-5$ & $5.88691 E-5$ & 0.000014159 & 0.0000617513 \\
\hline \multirow{5}{*}{$t=0.5$} & 0.1 & $1.1438 E-6$ & $1.1422 E-6$ & $3.36022 E-7$ & $1.14345 E-6$ \\
\hline & 0.2 & $3.9847 E-6$ & $3.9824 E-6$ & $1.17111 E-6$ & $3.98786 E-6$ \\
\hline & 0.3 & $1.38315 E-5$ & $1.38317 E-5$ & $4.06661 E-6$ & 0.0000138803 \\
\hline & 0.4 & $4.73792 E-5$ & $4.73753 E-5$ & 0.0000139399 & 0.0000479769 \\
\hline & 0.5 & $1.546009 E-4$ & $1.546023 E-4$ & 0.000045625 & 0.000161817 \\
\hline
\end{tabular}

$$
\begin{aligned}
V(0,2)= & -\left(8 \left(\operatorname{sech}^{4}(1-x+2 y)\right.\right. \\
& \left.\left.+2 \operatorname{sech}^{2}(1-x+2 y) \tanh (1-x+2 y)\right)\right) \\
& \times(\Gamma(1+2 \beta))^{-1} \\
U(2,0)= & \left(8 \operatorname{sech}^{2}(1-x+2 y)\right. \\
& \left.\times\left(2 \operatorname{sech}^{2}(1-x+2 y)+\tanh (1-x+2 y)\right)\right) \\
& \times(\Gamma(1+2 \alpha))^{-1} \\
V(1,1)= & \frac{8 \operatorname{sech}^{4}(1-x+2 y)}{\Gamma(1+\alpha+\beta)},
\end{aligned}
$$

and so on.

The approximate solutions, obtained in the series form, are given by

$$
\begin{aligned}
u(x, y, t) & =\sum_{k=0}^{\infty} \sum_{h=0}^{k} U(h, k-h) t^{(h \alpha+(k-h) \beta)} \\
& =U(0,0)+\sum_{k=1}^{\infty} \sum_{h=1}^{k} U(h, k-h) t^{(h \alpha+(k-h) \beta)}
\end{aligned}
$$

$$
\begin{aligned}
& =1-\tanh (-x+2 y+1)-\frac{2 t^{\alpha} \operatorname{sech}^{2}(1-x+2 y)}{\Gamma(1+\alpha)} \\
& -\frac{16 t^{\alpha+\beta} \operatorname{sech}^{4}(1-x+2 y)}{\Gamma(1+\alpha+\beta)} \\
& +\left(8 t^{2 \alpha} \operatorname{sech}^{2}(1-x+2 y)\right. \\
& \quad \times\left(2 \operatorname{sech}^{2}(1-x+2 y)\right. \\
& \left.\quad+\tanh ^{2}(1-x+2 y)\right) \\
& \left.\times(\Gamma(1+2 \alpha))^{-1}\right)+\cdots,
\end{aligned}
$$

$$
v(x, y, t)=\sum_{k=0}^{\infty} \sum_{h=0}^{k} V(h, k-h) t^{(h \alpha+(k-h) \beta)}
$$

$$
\begin{aligned}
= & V(0,0)+\sum_{k=1}^{\infty} \sum_{h=0}^{k} V(h, k-h) t^{(h \alpha+(k-h) \beta)} \\
= & 1+2 \tanh (1-x+2 y)+\frac{4 t^{\beta} \operatorname{sech}^{2}(1-x+2 y)}{\Gamma(1+\beta)} \\
& +\frac{8 t^{\alpha+\beta} \operatorname{sech}^{4}(1-x+2 y)}{\Gamma(1+\alpha+\beta)}
\end{aligned}
$$




$$
\begin{aligned}
&-\left(8 t^{2 \beta}(\right. \operatorname{sech}^{4}(1-x+2 y) \\
&+2 \operatorname{sech}^{2}(1-x+2 y) \\
&\times \tanh (1-x+2 y)) \\
&\left.\times(\Gamma(1+2 \beta))^{-1}\right)+\cdots
\end{aligned}
$$

When $\alpha=1$ and $\beta=1$, the solution in (36) becomes

$$
\begin{aligned}
u(x, y, t)= & 1-\tanh (-x+2 y+1)-2 t \operatorname{sech}^{2}(1-x+2 y) \\
& +4 t^{2} \operatorname{sech}^{2}(1-x+2 y) \\
& \times \tanh (1-x+2 y)+\cdots
\end{aligned}
$$

When $\alpha=1$ and $\beta=1$, the solution in (37) becomes

$$
\begin{aligned}
v(x, y, t) & \\
= & 1+2 \tanh (1-x+2 y)+4 t \operatorname{sech}^{2}(1-x+2 y) \\
& \quad-8 t^{2} \operatorname{sech}^{2}(1-x+2 y) \tanh (1-x+2 y)+\cdots .
\end{aligned}
$$

The solutions in (38) and (39) are exactly same as the Taylor series expansions of the exact solutions

$$
\begin{aligned}
u(x, y, t) & \\
= & 1-\tanh (-x+2 y+2 t+1) \\
= & 1-\tanh (-x+2 y+1)-2 t \operatorname{sech}^{2}(1-x+2 y) \\
& \quad+4 t^{2} \operatorname{sech}^{2}(1-x+2 y) \tanh (1-x+2 y)+\cdots \\
v(x, & y, t) \\
= & 1+2 \tanh (-x+2 y+2 t+1) \\
= & 1+2 \tanh ^{2}(1-x+2 y)+4 t \operatorname{sech}^{2}(1-x+2 y) \\
& \quad-8 t^{2} \operatorname{sech}^{2}(1-x+2 y) \tanh ^{2}(1-x+2 y)+\cdots .
\end{aligned}
$$

In order to verify whether the proposed methodology lead to higher accuracy, the numerical solutions have been evaluated with four terms for both CRFDTM and homotopy perturbation method (HPM). In case of $\alpha=1$ and $\beta=1$, Table 2 cites the comparison of results obtained in proposed CRFDTM with HPM when $y=0.3$. From these results we can certainly conclude that the proposed method CRFDTM provides remarkable accuracy in comparison to HPM.

Figure 1 explores the comparison of the numerical solutions of $u(x, y, t)$ and $v(x, y, t)$ for $\alpha=1, \beta=1$, and $t=0.3$ with the exact solutions in (40) and (41) respectively. Similarly, Figure 2 cites the comparison of the numerical solutions of $u(x, y, t)$ and $v(x, y, t)$ for $\alpha=1, \beta=1$, and $t=0.1$ with the exact solutions in (40) and (41) respectively. It can be observed that the numerical solutions obtained by the proposed method are exactly identical with the exact solution.

Figure 3 cites the numerical solutions of $u(x, y, t)$ and $v(x, y, t)$ for $\alpha=1 / 4, \beta=1 / 2$, and $t=0.3$, respectively, and Figure 4 depicts the numerical solutions of $u(x, y, t)$ and $v(x, y, t)$ for $\alpha=0.88, \beta=0.005$, and $t=0.1$ respectively. The figures show that when the value of $\alpha$ increases solution surface becomes flattened. On the other hand, when the value of $\beta$ decreases solution surface bifurcates into wave.

\section{Convergence Analysis and Error Estimate}

Theorem 6. Suppose that $D_{t}^{k \alpha} u(x, t) \in C([0, L] \times[0, T])$ for $k=0,1,2, \ldots, n+1$, where $0<\alpha<1$; then

$$
u(x, t) \cong \sum_{k=0}^{n} \sum_{h=0}^{k} U(h, k-h) t^{h \alpha+(k-h) \beta} .
$$

Moreover, there exist values $\xi_{1}, \xi_{2}$ where $0 \leq \xi_{1}, \xi_{2} \leq t$ so that the error term $E_{n}(x, t)$ has the form

$$
\begin{array}{r}
\left\|E_{n}(x, t)\right\|=\operatorname{Sup}_{\substack{0 \leq x \leq L \\
0 \leq t \leq T}}\left|\frac{D^{(n+1) \beta} u(x, 0+)}{\Gamma((n+1) \beta+1)} t^{(n+1) \beta}\right|, \\
\text { if } \xi_{1}, \xi_{2} \longrightarrow 0+.
\end{array}
$$

Proof. From Lemma 1, we have

$$
\begin{array}{r}
J^{\alpha} D^{\alpha} f(t)=f(t)-\sum_{k=0}^{m-1} \frac{t^{k}}{\Gamma(k+1)} f^{(k)}(0+), \\
m-1<\alpha<m .
\end{array}
$$

The error term is

$$
E_{n}(x, t)=u(x, t)-u^{*}(x, t),
$$

where

$$
\begin{gathered}
u(x, t)=\sum_{k=0}^{\infty} \sum_{h=0}^{k} \frac{D^{h \alpha+\beta(k-h)} u(x, 0)}{\Gamma(h \alpha+\beta(k-h)+1)} t^{h \alpha+\beta(k-h)}, \\
u^{*}(x, t)=\sum_{k=0}^{n} \sum_{h=0}^{k} \frac{D^{h \alpha+\beta(k-h)} u(x, 0)}{\Gamma(h \alpha+\beta(k-h)+1)} t^{h \alpha+\beta(k-h)} .
\end{gathered}
$$

Now, for $0<\alpha<1$,

$$
\begin{aligned}
& J^{h \alpha+\beta(k-h)} D^{h \alpha+\beta(k-h)} u(x, t) \\
&-J^{(h+1) \alpha+\beta(k-h)} D^{(h+1) \alpha+\beta(k-h)} u(x, t) \\
&= J^{h \alpha+\beta(k-h)} \\
& \times\left(D^{h \alpha+\beta(k-h)} u(x, t)-J^{\alpha} D^{\alpha}\left(D^{h \alpha+\beta(k-h)} u(x, t)\right)\right) \\
&= J^{h \alpha+\beta(k-h)} D^{h \alpha+\beta(k-h)} u(x, 0), \\
& \operatorname{since} \quad 0<\alpha<1, \mathrm{using} \quad(7) \\
&= \frac{D^{h \alpha+\beta(k-h)} u(x, 0)}{\Gamma(h \alpha+\beta(k-h)+1)} t^{h \alpha+\beta(k-h)} .
\end{aligned}
$$


TABLE 2: The comparison of results obtained in proposed method with HPM when $y=0.3, \alpha=1$, and $\beta=1$.

\begin{tabular}{|c|c|c|c|c|c|}
\hline & $t$ & $\left|u_{\text {Exact }}-u_{\text {HPM }}\right|$ & $\left|v_{\text {Exact }}-v_{\mathrm{HPM}}\right|$ & $\left|u_{\text {Exact }}-u_{\text {CRFDTM }}\right|$ & $\left|v_{\text {Exact }}-v_{\text {CRFDTM }}\right|$ \\
\hline \multirow{5}{*}{$x=0.1$} & 0.1 & 0.00388488 & 0.000409157 & 0.0000403885 & 0.000080777 \\
\hline & 0.2 & 0.0205864 & 0.00179639 & 0.00064308 & 0.00128616 \\
\hline & 0.3 & 0.0830004 & 0.00575008 & 0.00320473 & 0.00640947 \\
\hline & 0.4 & 0.192169 & 0.0154079 & 0.00989663 & 0.0197933 \\
\hline & 0.5 & 0.356304 & 0.0351083 & 0.0234905 & 0.046981 \\
\hline \multirow{5}{*}{$x=0.2$} & 0.1 & 0.00340343 & 0.000358137 & 0.0000377862 & 0.0000755724 \\
\hline & 0.2 & 0.0254938 & 0.000618968 & 0.000619573 & 0.00123915 \\
\hline & 0.3 & 0.0946681 & 0.00097818 & 0.00315603 & 0.00631207 \\
\hline & 0.4 & 0.211242 & 0.00333882 & 0.00991152 & 0.019823 \\
\hline & 0.5 & 0.381651 & 0.0109793 & 0.0238381 & 0.0476761 \\
\hline \multirow{5}{*}{$x=0.3$} & 0.1 & 0.00290734 & 0.000169508 & 0.0000309013 & 0.0000618027 \\
\hline & 0.2 & 0.0273013 & 0.00190188 & 0.000536002 & 0.001072 \\
\hline & 0.3 & 0.0924439 & 0.0086713 & 0.00284027 & 0.00568053 \\
\hline & 0.4 & 0.193425 & 0.0207692 & 0.00918199 & 0.018364 \\
\hline & 0.5 & 0.330375 & 0.0372769 & 0.0225741 & 0.0451482 \\
\hline \multirow{5}{*}{$x=0.4$} & 0.1 & 0.00271456 & 0.000255194 & 0.0000181808 & 0.0000363616 \\
\hline & 0.2 & 0.0220717 & 0.00656152 & 0.000368845 & 0.00073769 \\
\hline & 0.3 & 0.0620409 & 0.0259301 & 0.00214616 & 0.00429232 \\
\hline & 0.4 & 0.103938 & 0.0634979 & 0.00738142 & 0.0147628 \\
\hline & 0.5 & 0.133654 & 0.122694 & 0.0189596 & 0.0379192 \\
\hline \multirow{5}{*}{$x=0.5$} & 0.1 & 0.0033497 & 0.00104781 & $1.95074 E-6$ & $3.90148 E-6$ \\
\hline & 0.2 & 0.00408341 & 0.0143686 & 0.0000931316 & 0.000186263 \\
\hline & 0.3 & 0.0167561 & 0.0541981 & 0.000951695 & 0.00190339 \\
\hline & 0.4 & 0.105827 & 0.132966 & 0.00414311 & 0.00828621 \\
\hline & 0.5 & 0.304 & 0.261277 & 0.0121501 & 0.0243001 \\
\hline
\end{tabular}

The $n$th order approximation for $u(x, t)$ is

$$
\begin{aligned}
u^{*}(x, t)= & \sum_{k=0}^{n} \sum_{h=0}^{k} \frac{D^{h \alpha+\beta(k-h)} u(x, 0)}{\Gamma(h \alpha+\beta(k-h)+1)} t^{h \alpha+\beta(k-h)} \\
= & \sum_{k=0}^{n} \sum_{h=0}^{k}\left(J^{h \alpha+\beta(k-h)} D^{h \alpha+\beta(k-h)} u(x, t)\right. \\
& \left.-J^{(h+1) \alpha+\beta(k-h)} D^{(h+1) \alpha+\beta(k-h)} u(x, t)\right), \\
= & \sum_{k=0}^{n} J^{k \beta} D^{k \beta} u(x, t) \\
& -\sum_{h=0}^{n} J^{(h+1) \alpha+\beta(n-h)} D^{(h+1) \alpha+\beta(n-h)} u(x, t) \\
= & u(x, t)+\sum_{k=0}^{n-1} J^{(k+1) \beta} D^{(k+1) \beta} u(x, t) \\
& -\sum_{h=0}^{n} J^{(h+1) \alpha+\beta(n-h)} D^{(h+1) \alpha+\beta(n-h)} u(x, t) .
\end{aligned}
$$

Therefore, from (48), the error term becomes

$$
\begin{aligned}
E_{n}(x, t)= & u(x, t)-u^{*}(x, t) \\
= & \sum_{h=0}^{n} J^{(h+1) \alpha+\beta(n-h)} D^{(h+1) \alpha+\beta(n-h)} u(x, t) \\
& -\sum_{k=0}^{n-1} J^{(k+1) \beta} D^{(k+1) \beta} u(x, t) \\
= & \sum_{i=0}^{n} J^{(i+1) \alpha+\beta(n-i)} D^{(i+1) \alpha+\beta(n-i)} u(x, t) \\
& -\sum_{i=0}^{n-1} J^{(i+1) \beta} D^{(i+1) \beta} u(x, t) \\
= & \sum_{i=0}^{n} \frac{1}{\Gamma((i+1) \alpha+\beta(n-i))} \\
& \times \int_{0}^{t}(t-\tau)^{(i+1) \alpha+\beta(n-i)-1} D^{(i+1) \alpha+\beta(n-i)} u(x, \tau) d \tau \\
& -\sum_{i=0}^{n-1} \frac{1}{\Gamma((i+1) \beta)}
\end{aligned}
$$




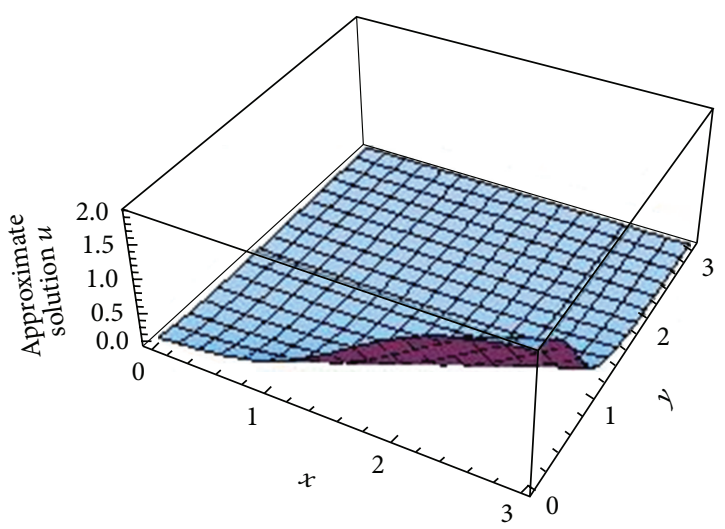

(a)

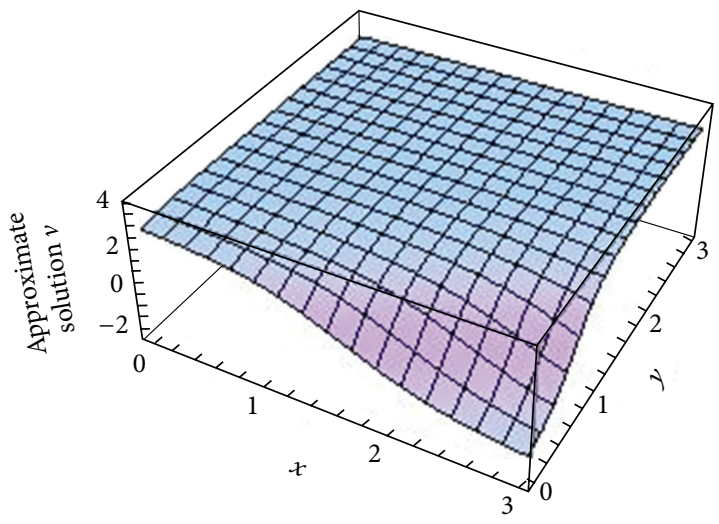

(c)

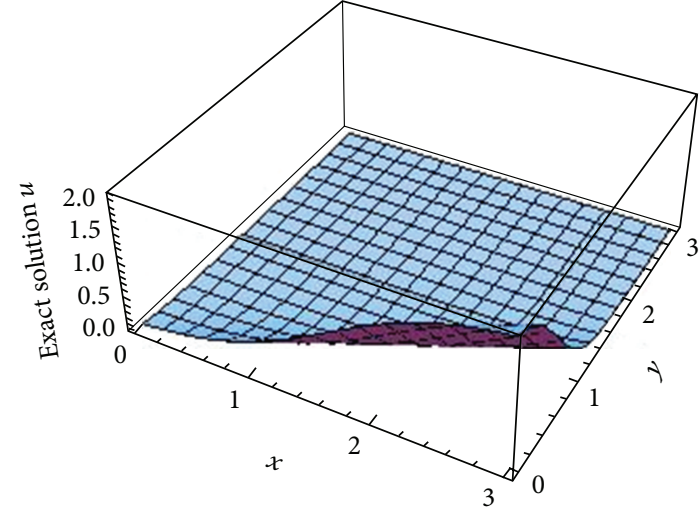

(b)

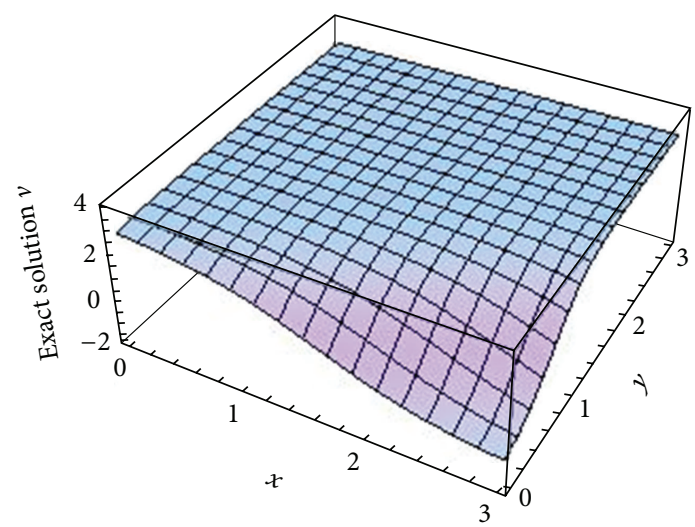

(d)

Figure 1: The surfaces show (a) the numerical approximate solution of $u(x, y, 0.3)$, (b) the exact solution of $u(x, y, 0.3)$, (c) the numerical approximate solution of $v(x, y, 0.3)$, and (d) the exact solution of $v(x, y, 0.3)$ when $\alpha=1$ and $\beta=1$.

$$
\begin{gathered}
\times \int_{0}^{t}(t-\tau)^{(i+1) \beta-1} D^{(i+1) \beta} u(x, \tau) d \tau \\
=\sum_{i=0}^{n} \frac{D^{(i+1) \alpha+\beta(n-i)} u\left(x, \xi_{1}\right)}{\Gamma((i+1) \alpha+\beta(n-i)+1)} t^{(i+1) \alpha+\beta(n-i)} \\
-\sum_{i=0}^{n-1} \frac{D^{(i+1) \beta} u\left(x, \xi_{2}\right)}{\Gamma((i+1) \beta+1)} t^{(i+1) \beta},
\end{gathered}
$$

applying integral mean value theorem

$$
\begin{aligned}
= & \sum_{i=0}^{n-1} \frac{D^{(i+1) \alpha+\beta(n-i)} u\left(x, \xi_{1}\right)}{\Gamma((i+1) \alpha+\beta(n-i)+1)} t^{(i+1) \alpha+\beta(n-i)} \\
& +\frac{D^{(n+1) \alpha} u\left(x, \xi_{1}\right)}{\Gamma((n+1) \alpha+1)} t^{(n+1) \alpha} \\
& -\sum_{i=0}^{n-1} \frac{D^{(i+1) \beta} u\left(x, \xi_{2}\right)}{\Gamma((i+1) \beta+1)} t^{(i+1) \beta} \\
= & \sum_{i=0}^{n-1}\left[\frac{D^{(i+1) \alpha+\beta(n-i)} u\left(x, \xi_{1}\right)}{\Gamma((i+1) \alpha+\beta(n-i)+1)} t^{(i+1) \alpha+\beta(n-i)}\right.
\end{aligned}
$$

$$
\begin{gathered}
\left.-\frac{D^{(i+1) \beta} u\left(x, \xi_{2}\right)}{\Gamma((i+1) \beta+1)} t^{(i+1) \beta}\right] \\
+\frac{D^{(n+1) \alpha} u\left(x, \xi_{1}\right)}{\Gamma((n+1) \alpha+1)} t^{(n+1) \alpha} .
\end{gathered}
$$

Using generalized Taylor's series formula (8), (49) becomes

$$
\begin{aligned}
E_{n}(x, t)= & u(x, t)-\frac{D^{(n+1) \alpha} u\left(x, \zeta_{1}\right)}{\Gamma((n+1) \alpha+1)} t^{(n+1) \alpha}-u(x, t) \\
& +\frac{D^{(n+1) \beta} u\left(x, \zeta_{2}\right)}{\Gamma((n+1) \beta+1)} t^{(n+1) \beta} \\
& +\frac{D^{(n+1) \alpha} u\left(x, \xi_{1}\right)}{\Gamma((n+1) \alpha+1)} t^{(n+1) \alpha}
\end{aligned}
$$

where $0 \leq \zeta_{1}, \zeta_{2} \leq \max \left\{\xi_{1}, \xi_{2}\right\}, \xi_{1}, \xi_{2} \rightarrow 0+$. 


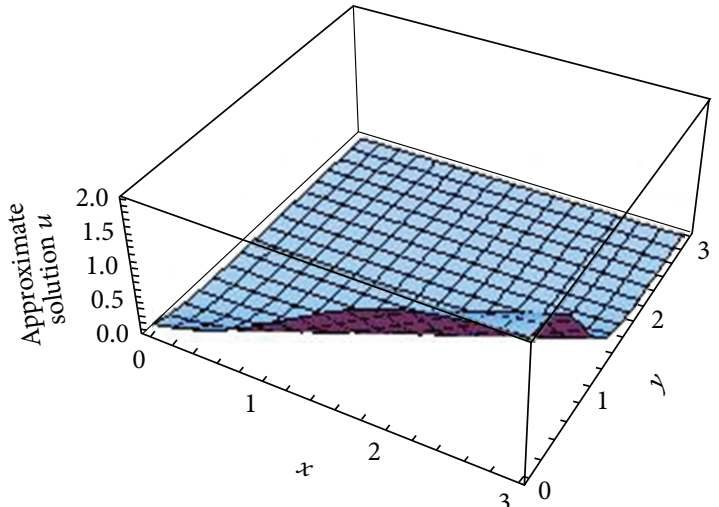

(a)

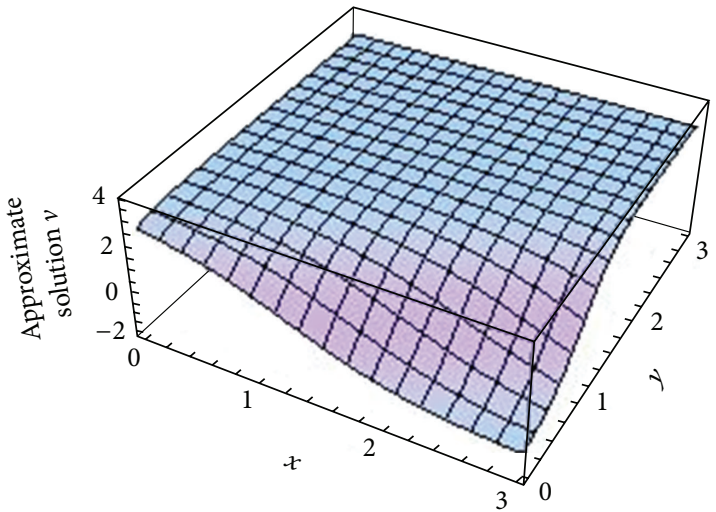

(c)

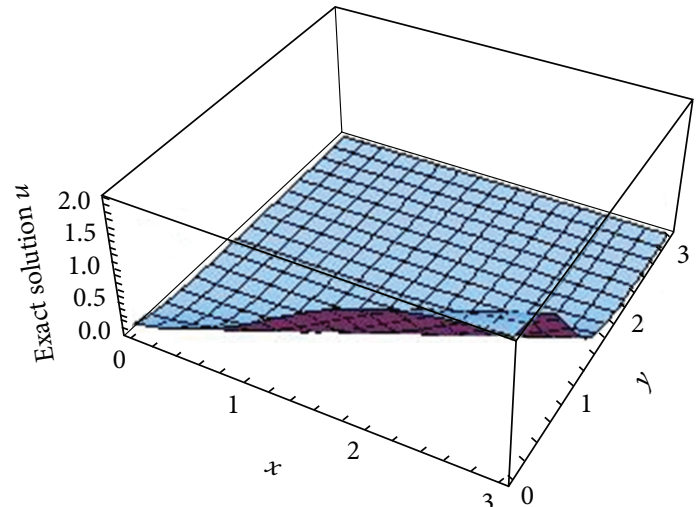

(b)

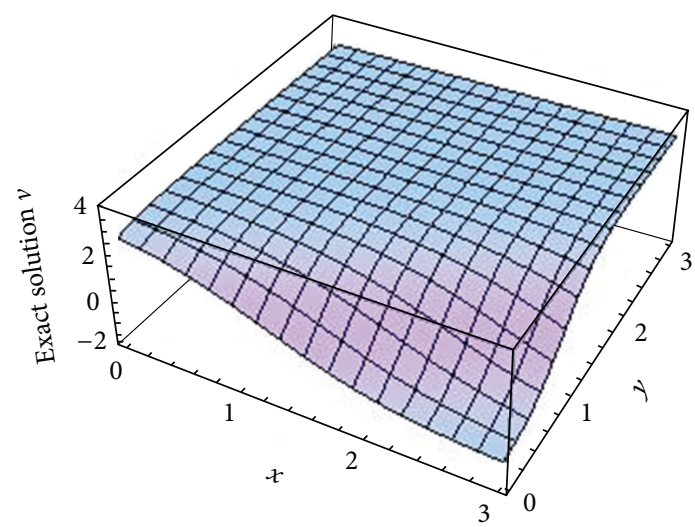

(d)

FiguRE 2: The surfaces show (a) the numerical approximate solution of $u(x, y, 0.1)$, (b) the exact solution of $u(x, y, 0.1)$, (c) the numerical approximate solution of $v(x, y, 0.1)$, and (d) the exact solution of $v(x, y, 0.1)$ when $\alpha=1$ and $\beta=1$.

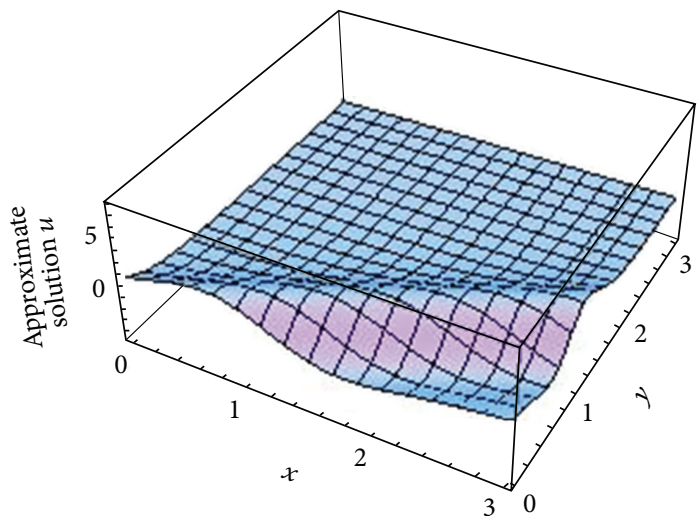

(a)

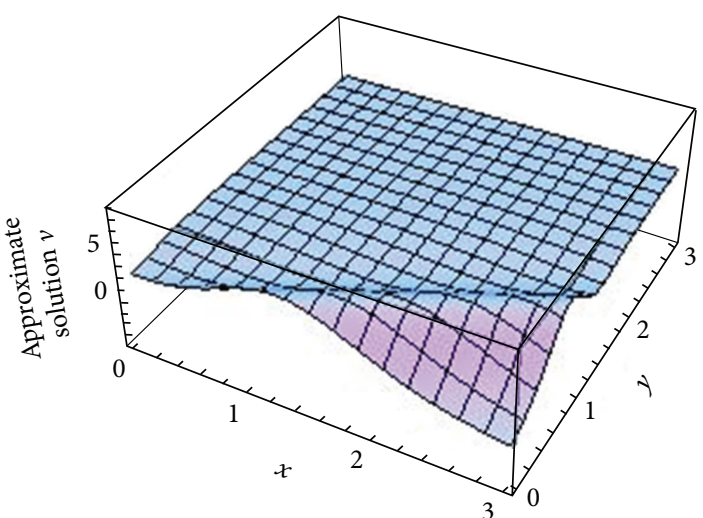

(b)

FIGURE 3: The surfaces show (a) the numerical approximate solution of $u(x, y, 0.3)$ and (b) the numerical approximate solution of $v(x, y, 0.3)$ when $\alpha=1 / 4$ and $\beta=1 / 2$.

This implies

$$
\begin{aligned}
\left\|E_{n}\right\| & =\left\|u(x, t)-u^{*}(x, t)\right\| \\
& =\operatorname{Sup}_{\substack{0 \leq x \leq L \\
0 \leq t \leq T}} \mid \frac{D^{(n+1) \beta} u\left(x, \zeta_{2}\right)}{\Gamma((n+1) \beta+1)} t^{(n+1) \beta}
\end{aligned}
$$

$$
\begin{aligned}
& -\frac{D^{(n+1) \alpha} u\left(x, \zeta_{1}\right)}{\Gamma((n+1) \alpha+1)} t^{(n+1) \alpha} \\
& +\frac{D^{(n+1) \alpha} u\left(x, \xi_{1}\right)}{\Gamma((n+1) \alpha+1)} t^{(n+1) \alpha} \mid<\infty
\end{aligned}
$$




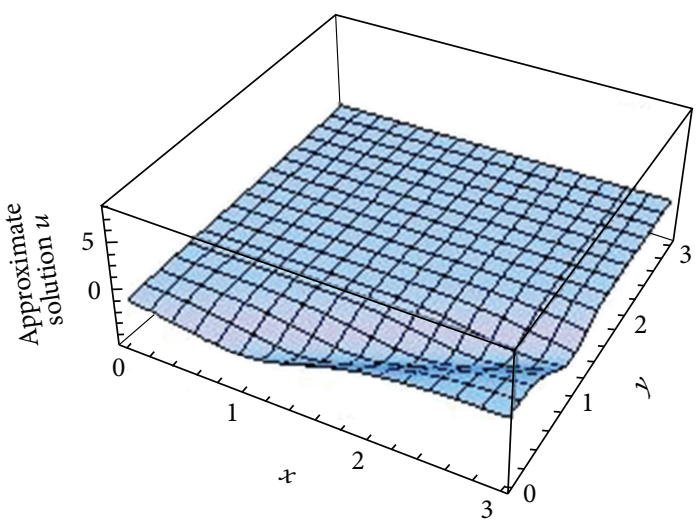

(a)

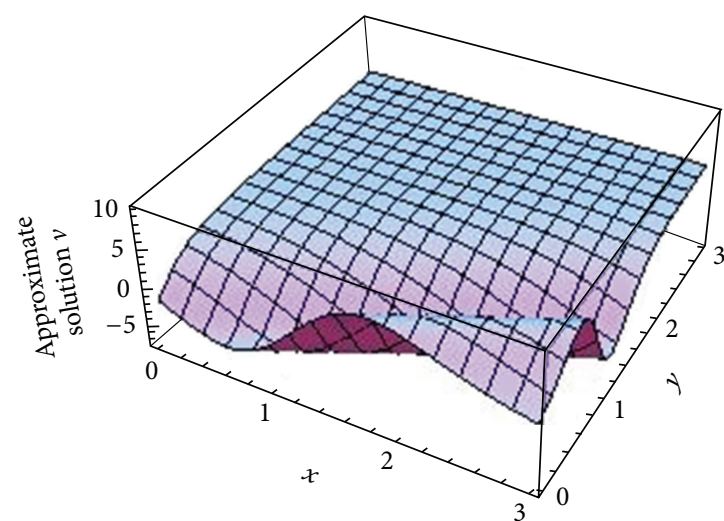

(b)

FIGURE 4: The surfaces show (a) the numerical approximate solution of $u(x, y, 0.1)$ and (b) the numerical approximate solution of $v(x, y, 0.1)$ when $\alpha=0.88$ and $\beta=0.005$.

$$
\begin{array}{r}
=\operatorname{Sup}_{\substack{0 \leq x \leq L \\
0 \leq t \leq T}}\left|\frac{D^{(n+1) \beta} u(x, 0+)}{\Gamma((n+1) \beta+1)} t^{(n+1) \beta}\right|, \\
\text { since } \xi_{1}, \xi_{2} \longrightarrow 0+.
\end{array}
$$

As $n \rightarrow \infty,\left\|E_{n}\right\| \rightarrow 0$.

Hence, $u(x, t)$ can be approximated as

$$
\begin{aligned}
u(x, t) & =\sum_{k=0}^{\infty} \sum_{h=0}^{k} U(h, k-h) t^{h \alpha+(k-h) \beta} \\
& \cong \sum_{k=0}^{n} \sum_{h=0}^{k} U(h, k-h) t^{h \alpha+(k-h) \beta},
\end{aligned}
$$

with the error term given in (51).

\section{Conclusion}

In this paper, a new approximate numerical technique, coupled fractional reduced differential transform, has been proposed for solving nonlinear fractional partial differential equations. The proposed method is only well suited for coupled fractional linear and nonlinear differential equations. In comparison to other analytical methods, the present method is an efficient tool to determine approximate solution of nonlinear coupled fractional partial differential equations. The obtained results demonstrate the reliability of the proposed algorithm and its applicability to nonlinear coupled fractional evolution equations. It also exhibits that the proposed method is very efficient and powerful technique in finding the solutions of the nonlinear coupled time fractional differential equations. The main advantage of the proposed method is that it requires less amount of computational overhead in comparison to other numerical and analytical approximate methods and consequently introduces a significant improvement in solving coupled fractional nonlinear equations over existing methods available in open literature. The application of the proposed method for the solutions of time fractional coupled $(2+1)$ Burger equations satisfactorily justifies its simplicity and efficiency.

\section{Appendix}

Proof of Theorem 3 (iii). One has

$$
\begin{aligned}
f(x, y, t)= & u(x, y, t) v(x, y, t) \\
= & \left(\sum_{k=0}^{\infty} \sum_{h=0}^{k} U(h, k-h) t^{h \boldsymbol{\alpha}+(k-h) \boldsymbol{\beta}}\right) \\
& \times\left(\sum_{k=0}^{\infty} \sum_{h=0}^{k} V(h, k-h) t^{h \boldsymbol{\alpha}+(k-h) \boldsymbol{\beta}}\right) \\
= & U(0,0) V(0,0) \\
& +(U(1,0) V(0,0)+U(0,0) V(1,0)) t^{\boldsymbol{\alpha}} \\
& +(U(0,1) V(0,0)+U(0,0) V(0,1)) t^{\boldsymbol{\beta}} \\
& +(U(1,0) V(0,1)+U(0,1) V(1,0) \\
& +\cdots \\
& +\sum_{k=0}^{\infty} \sum_{h=0}^{k}\left(\sum_{l=0}^{h} \sum_{s=0}^{k-h} U(h-l, s) V(l, k-h-s)\right) \\
& \times t^{h \boldsymbol{\alpha}+(k-h) \boldsymbol{\beta}} .
\end{aligned}
$$

Hence, $F(h, k-h)=\sum_{l=0}^{h} \sum_{s=0}^{k-h} U(h-l, s) V(l, k-h-s)$. 


\section{Conflict of Interests}

The author declares that there is no conflict of interests regarding the publication of this paper.

\section{Acknowledgment}

The author would like to express his sincere thanks and gratitude to the anonymous learned reviewers for their kind suggestions for the betterment and improvement of the present paper.

\section{References}

[1] I. Podlubny, Fractional Differential Equations, vol. 198 of Mathematics in Science and Engineering, Academic Press, New York, NY, USA, 1999.

[2] R. Hilfer, Applications of Fractional Calculus in Physics, World Scientific, Singapore, 2000.

[3] S. S. Ray and R. K. Bera, "Analytical solution of a dynamic system containing fractional derivative of order one-half by Adomian decomposition method," Transaction ASME Journal of Applied Mechanics, vol. 72, no. 2, pp. 290-295, 2005.

[4] S. S. Ray and R. K. Bera, "An approximate solution of a nonlinear fractional differential equation by Adomian decomposition method," Applied Mathematics and Computation, vol. 167, no. 1, pp. 561-571, 2005.

[5] S. Saha Ray and R. K. Bera, "Analytical solution of a fractional diffusion equation by Adomian decomposition method," Applied Mathematics and Computation, vol. 174, no. 1, pp. 329336, 2006.

[6] S. S. Ray, "Exact solutions for time-fractional diffusion-wave equations by decomposition method," Physica Scripta, vol. 75, no. 1, article 008, pp. 53-61, 2007.

[7] S. S. Ray, "A new approach for the application of Adomian decomposition method for the solution of fractional space diffusion equation with insulated ends," Applied Mathematics and Computation, vol. 202, no. 2, pp. 544-549, 2008.

[8] S. S. Ray and R. K. Bera, "Analytical solution of the Bagley Torvik equation by Adomian decomposition method," Applied Mathematics and Computation, vol. 168, no. 1, pp. 398-410, 2005.

[9] S. Saha Ray, "On Haar wavelet operational matrix of general order and its application for the numerical solution of fractional Bagley Torvik equation," Applied Mathematics and Computation, vol. 218, no. 9, pp. 5239-5248, 2012.

[10] S. S. Ray and A. Patra, "An explicit finite difference scheme for numerical solution of fractional neutron point kinetic equation," Annals of Nuclear Energy, vol. 41, pp. 61-66, 2012.

[11] J. Nee and J. Duan, "Limit set of trajectories of the coupled viscous Burgers' equations," Applied Mathematics Letters, vol. 11, no. 1, pp. 57-61, 1998.

[12] M. Caputo, "Linear models of dissipation whose Q is almost frequency independent-part II," Royal Astronomical Society, vol. 13, p. 529, 1967.

[13] M. Caputo, Elasticità e Dissipazione, Zanichelli, Bologna, Italy, 1969.

[14] Z. M. Odibat and N. T. Shawagfeh, "Generalized Taylor's formula," Applied Mathematics and Computation, vol. 186, no. 1, pp. 286-293, 2007.
[15] A. A. Soliman, "On the solution of two-dimensional coupled Burgers' equations by variational iteration method," Chaos, Solitons and Fractals, vol. 40, no. 3, pp. 1146-1155, 2009.

[16] E. Hızela and S. Küçükarslan, "Homotopy perturbation method for (2+1)-dimensional coupled Burgers system," Nonlinear Analysis: Real World Applications, vol. 10, pp. 1932-1938, 2009. 

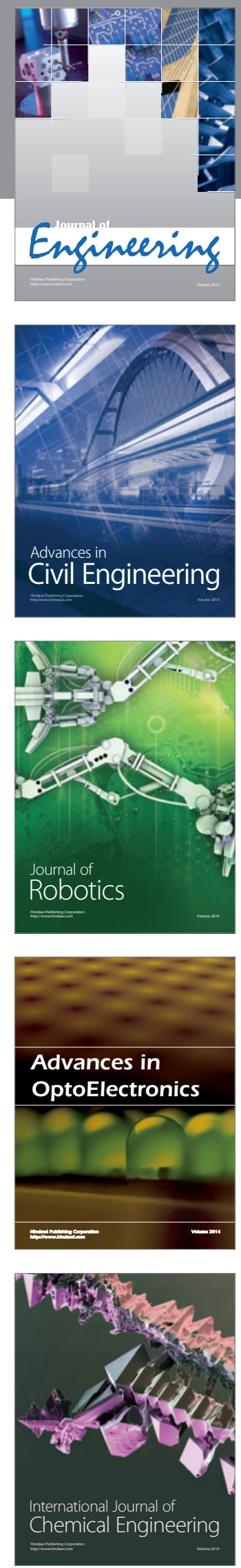

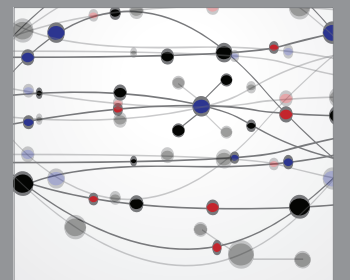

The Scientific World Journal
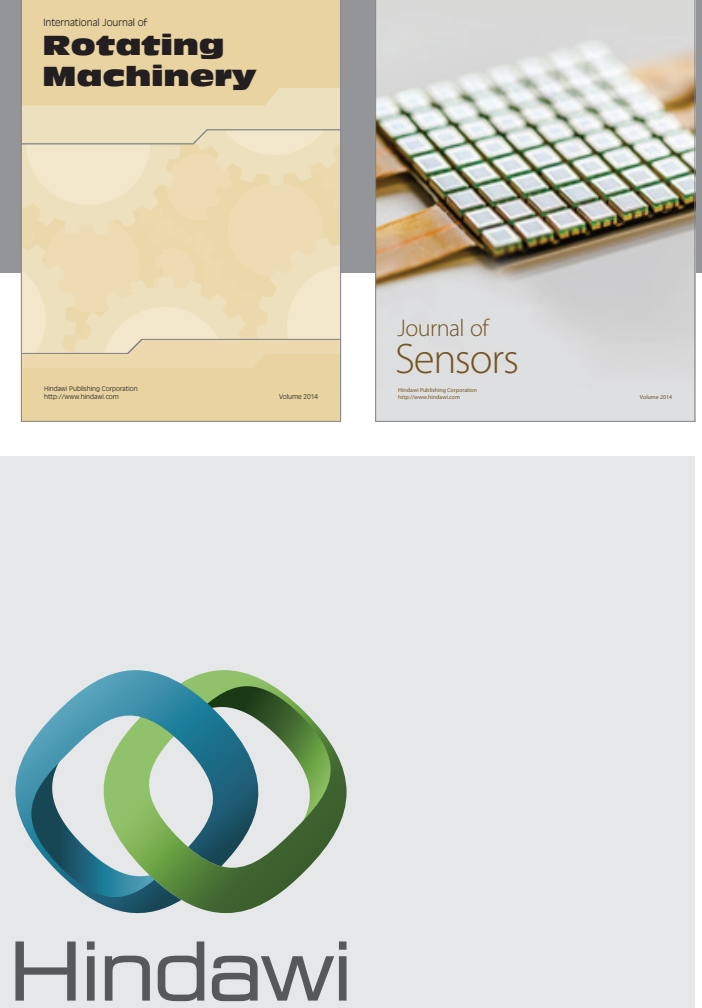

Submit your manuscripts at http://www.hindawi.com
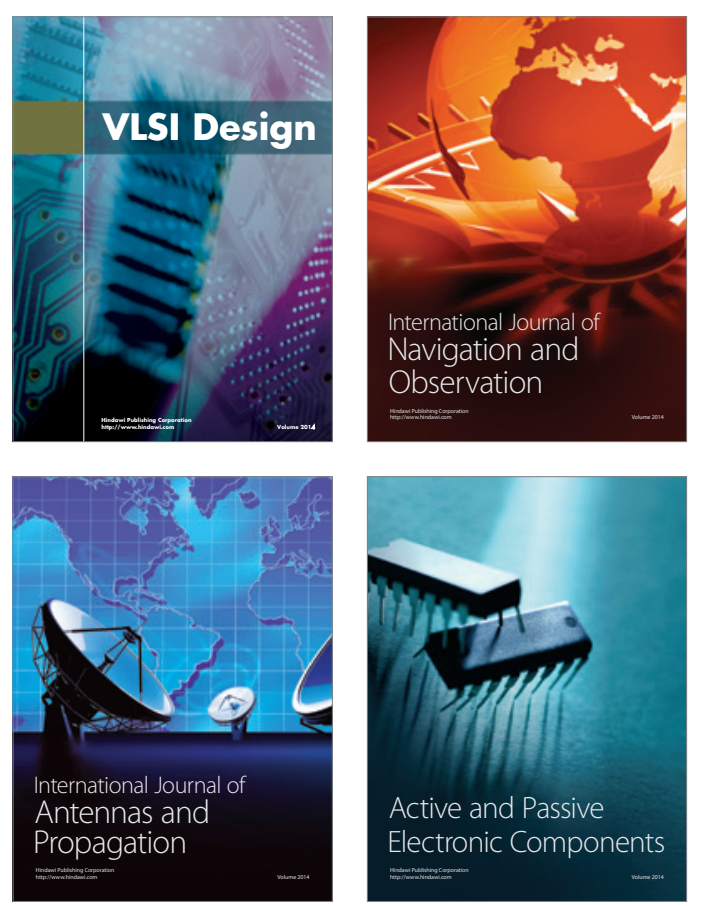
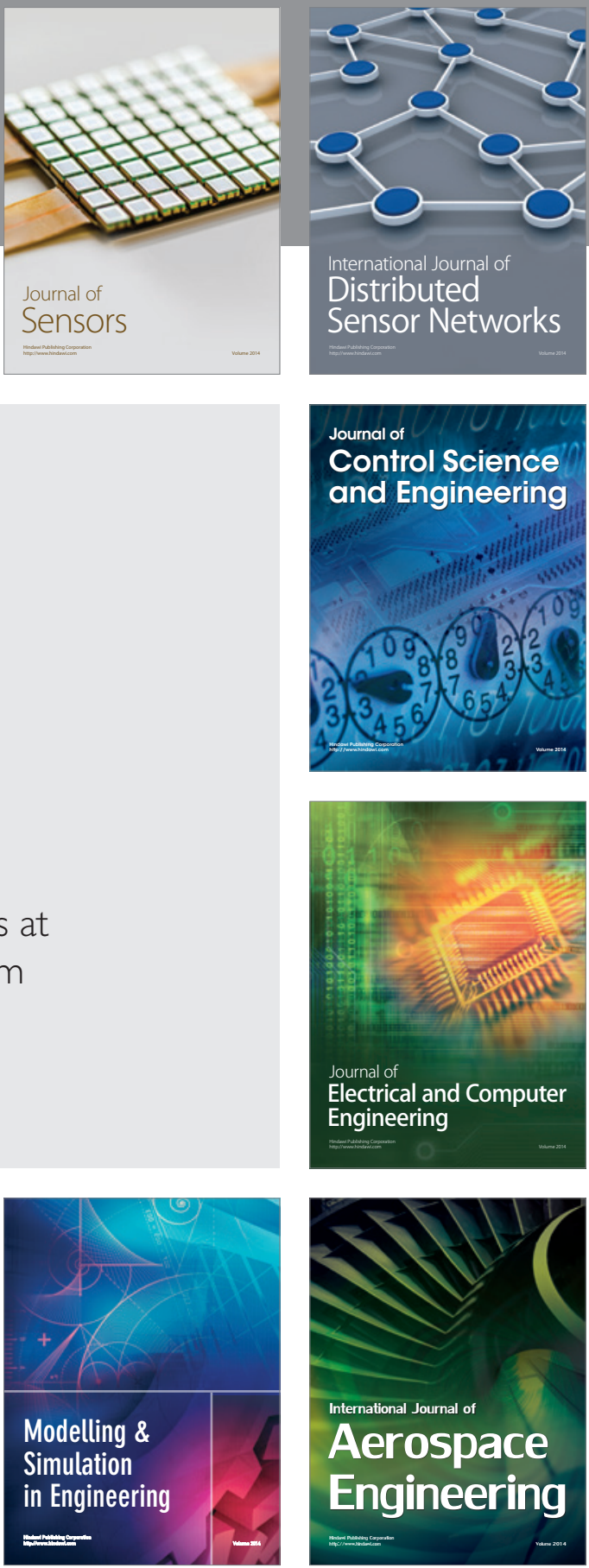

Journal of

Control Science

and Engineering
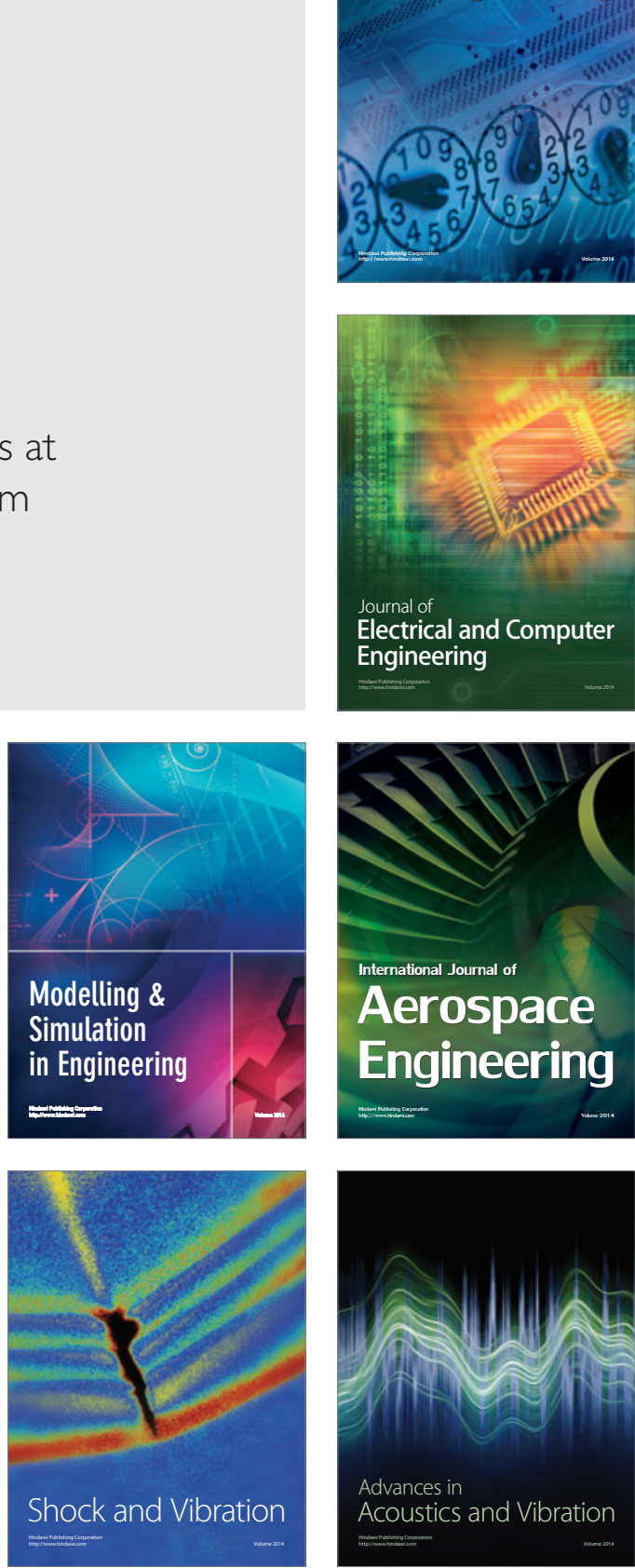